\title{
ON THE UNIQUENESS OF SOLITARY ROSSBY WAVES
}

\author{
P. E. KLOEDEN ${ }^{1}$
}

(Received 18 December 1985; revised 17 March 1986 and 10 June 1986)

\begin{abstract}
The explicit solitary Rossby wave solutions found by Larichev, Reznik and Berestov are shown to be unique for the model equations considered, in the sense that there are no other antisymmetric wave solutions which are not of these forms. This is done by adapting arguments used by Amick and Fraenkel to show the uniqueness of the Hill's vortex solution. It is based on the maximum principle and the domain folding method of Gidas, $\mathrm{Ni}$ and Nirenberg, and involves showing that the function $\psi / y$ is radially symmetric, where $\psi$ is the streamfunction of a solitary wave and $y$ the horizontal cartesian coordinate perpendicular to the $x$-axis, along which the waves move at steady positive speed. This argument is also used to show the uniqueness of the well-known explicit solutions for cylindrical vortices. The result does not apply directly to rider solutions of Flierl et al., which are not antisymmetric, but it does restrict the possible rider solutions that can form because of their association with particular antisymmetric solutions.
\end{abstract}

\section{Introduction}

Explicit formulae for two- and three-dimensional solitary Rossby waves have been determined by Larichev and Reznik [7] for a barotropic ocean and by Berestov [2] for a baroclinic ocean. These are exact solutions of the nonlinear quasi-geostrophic vorticity equations in the $\beta$-plane approximation ([3], [8]). The waves are damped exponentially at large distances from their centres, which move at constant speed along the equator, and are antisymmetric about the equator. In deriving these solutions the authors assumed that the switch over from one form of the equations to another occurs on circles about the centre of the wave. Then they used the standard applied mathematical technique of separating variables,

\footnotetext{
${ }^{1}$ School of Mathematical and Physical Sciences, Murdoch University, Murdoch, W. A. 6150; and Centre for Environmental Fluid Dynamics, University of W. A., Nedlands, W. A. 6009, Australia. (C) Copyright Australian Mathematical Society 1987, Serial-fee code 0334-2700/87
} 
specifically of writing the streamfunction $\psi$ as the product of an unknown function of the radial variable with the sines of the polar or spherical coordinate angles. In this way they reduced the problem to one of solving Bessel-like equations for the radial function.

The purpose of this note is to verify that this assumption and intuitive procedure do in fact generate all such antisymmetric solitary Rossby wave solutions for the given model equations, that is to establish the uniqueness of the above explicit solutions within the class of antisymmetric solutions. This is done by using the maximum principle and adapting the argument used by Amick and Fraenkel [1] to prove the uniqueness of Hill's vortex ring solution. It in turn is based on the domain folding method of Gidas, Ni and Nirenberg [4] and involves showing the radial symmetry of the function $\psi / y$, where $x$ and $y$ are horizontal Cartesian coordinates with the $x$-axis translated at constant positive speed $U$, so that the wave centre is always at the origin.

The basic equations and Berestov's solutions are stated in Section 2. The separability of the three-dimensional solutions is then justified in Section 3 by applying a theorem of Amick and Fraenkel to show that the function $Z=-\psi / y$ for $y>0$, considered as a function on $\mathbf{R}^{5}$ with Cartesian coordinates $x_{1}=x$, $x_{2}=z$ and spherical coordinates $y=\sqrt{x_{3}^{2}+x_{4}^{2}+x_{5}^{2}}, \theta$ and $\phi(Z$ being independent of $\theta$ and $\phi$ ), is radially symmetric. To do this we first show that $Z$ is contained in the Sobolev space $W^{1,2}\left(\mathbf{R}^{5}\right)$ and is strictly positive. The uniqueness of Berestov's solitary wave solutions is then concluded in Section 4. It must be emphasized that this uniqueness is in fact for a family of similar solutions, rather than one specific solution. Moreover it is for the specific model equations considered and for antisymmetric solutions only. (The rider solutions of Flierl et al [3] consequently do not come within the scope of this result, though since rider solutions are always associated with antisymmetric solutions the result indirectly restricts the types of rider solution that can form.) An analogous argument yields the uniqueness of the two-dimensional solitary Rossby wave solutions of Larichev and Reznik and also, as is briefly explained in Section 5, the uniqueness of the well-known explicit solutions for cylindrical vortices.

\section{Basic equations and Berestov's solutions}

In the $\beta$-plane approximation, the quasi-geostrophic potential vorticity equation is

$$
\left(\frac{\partial}{\partial t}-\frac{\partial \psi}{\partial y} \cdot \frac{\partial}{\partial x}+\frac{\partial \psi}{\partial x} \cdot \frac{\partial}{\partial y}\right)\left(\frac{\partial^{2} \psi}{\partial x^{2}}+\frac{\partial^{2} \psi}{\partial y^{2}}+\frac{\partial}{\partial z}\left(\frac{f_{0}^{2}}{N_{0}^{2}} \frac{\partial \psi}{\partial z}\right)+\beta y\right)=0
$$


where $\psi$ is the quasi-geostrophic streamfunction, $N_{0}$ the Brunt-Väisäla frequency and $f=f_{0}+\beta y$ the Coriolis parameter. Here $N_{0}, f_{0}$ and $\beta$ are assumed positive and constant.

Stationary wave solutions of (2.1) propagating along the $x$-axis with constant positive speed $U$ have the form $\psi=\psi(x-U t, y, z)$ and satisfy the equation

$$
J\left(\psi+U y^{\prime}, \Delta_{3} \psi+\beta y^{\prime}\right)=0,
$$

where $J(f, g)=\partial f / \partial x^{\prime} \partial g / \partial y^{\prime}-\partial f / \partial y^{\prime} \partial g / \partial x^{\prime}$ in terms of the new coordinates $x^{\prime}=x-U t, y^{\prime}=y, z^{\prime}=N_{0} z / f_{0}$ (the primes will henceforth be omitted) and $\Delta_{3}$ is the three-dimensional Laplacian. The general solution of (2.2) satisfies

$$
\Delta_{3} \psi+\beta y=F(\psi+U y),
$$

where $F$ is an arbitrary function of appropriate smoothness.

Berestov [2] sought solutions $\psi$ of (2.3) which were twice continuously differentiable and which, with their first-order derivatives, decreased exponentially to zero as $r^{2}=x^{2}+y^{2}+z^{2} \rightarrow \infty$. With solitary waves symmetric about the $x$-axis, or equivalently with $\psi$ an odd function in $y$, he essentially restricted attention to the half-space $y \geqslant 0$ and considered the piecewise linear mapping $F$ defined by

$$
F(\zeta)= \begin{cases}-k^{2} \zeta & \text { for } \zeta \leqslant 0 \\ p^{2} \zeta & \text { for } \zeta \geqslant 0\end{cases}
$$

where $k$ and $p$ are positive parameters to be determined. Berestov introduced spherical coordinates $r, \theta$ and $\phi$ (so that $x=r \sin \theta \cos \phi, y=r \sin \theta \sin \phi$ and $z=r \cos \theta$ ), separated variables

$$
\psi(r, \theta, \phi)=\Phi(r, \theta) \sin \phi
$$

and got

$$
L \Phi+\beta r \sin \theta=F(\Phi+U r \sin \theta)
$$

where

$$
L \Phi=\frac{1}{r^{2}} \frac{\partial}{\partial r}\left(r^{2} \frac{\partial \Phi}{\partial r}\right)+\frac{1}{r^{2} \sin \theta} \frac{\partial}{\partial \theta}\left(\sin \theta \frac{\partial \Phi}{\partial \theta}\right)-\frac{1}{r^{2} \sin ^{2} \theta} \Phi
$$

under the assumption that $\Phi+U r \sin \theta<0$ for $r<a$ and $>0$ for $r>a$ for some positive parameter $a$ to be determined. He then separated variables again

$$
\Phi(r, \theta)=R(r) \sin \theta
$$


solved Bessel-like ordinary differential equations in $R$ to get

$$
R(r)= \begin{cases}A r^{-1 / 2} J_{3 / 2}(k r)-\frac{U k^{2}+\beta}{k^{2}} r & \text { for } r<a \\ B r^{-1 / 2} K_{3 / 2}(p r) & \text { for } r>a,\end{cases}
$$

and evaluated $p^{2}=\beta / U$ from the assumption that $\psi$ vanished for $r \rightarrow \infty$. Finally, by matching solutions and their radial derivatives at $r=a$, where he assumed $\Phi+U r \sin \theta$ changed sign, Berestov found explicit negative values for $A$ and $B$, and showed that $k a$ took a unique positive value. See [2] for additional details.

The piecewise linear mapping (2.4) is somewhat artificial and appears to have been chosen to enable the variables to be separated. On the other hand, the requirement that $\psi$ and its derivatives vanish as $r \rightarrow \infty$ means that the functions $F$ in (2.3) must be at least asymptotically linear with $\beta=F(U)$.

The two-dimensional solutions of Larichev and Reznik [7] were derived in a similar way, essentially with the $z$ and $\theta$ variables omitted above and with the Bessel and modified Bessel functions of order 1 instead of $3 / 2$. Berestov called such solutions 2-layer solitary waves. He also considered 3-layer waves in both two and three dimensions, for which the first part of $F$ in (2.4) holds for $a<r<b$ and the second part for both $r<a$ and $r>b$. Here $b$ is an additional parameter determined in the same way as $a$. Berestov commented that it was not generally possible to construct solutions with more than three layers by this procedure as it led to an overdetermined system involving more equations than unknown parameters.

The mapping $F$ in (2.3) was not expressed quite in the same way as (2.4) by Berestov. Rather he assumed that it had one linear form for $r<a$ and the other linear form for $r>a$, so his mapping $F$ depended on $r$ as well as on $\psi+U y$, which is not strictly speaking mathematically correct. With the restriction to antisymmetric solutions, like those found by Berestov, our functional form (2.4) is mathematically correct and equivalent to Berestov's formulation. This restriction to antisymmetric solutions does however exclude from consideration the rider solutions of Flierl et al. [3].

\section{Separability of solutions}

In deriving his formulae for two- and three-layer solitary Rossby waves Berestov assumed that he could separate the solutions of (2.3) in the form (2.5) to (2.8). It will be shown here that every antisymmetric solution with at least a modest amount of regularity can be separated in this way. 
Let $\Pi=\left\{(x, y, z) \in \mathbf{R}^{3} ; y>0\right\}$. A function $\psi$ on $\Pi$ will be called a weak solution of the partial differential equation (2.3), with $F$ as defined by (2.4), if it belongs to the Sobolev space $W^{1,2}(\Pi)$ with

$$
\psi^{2} / y \rightarrow 0 \text { as } y \rightarrow 0^{+}
$$

for almost all $(x, z) \in \mathbf{R}^{2}$ and satisfies (2.3) in the distributional sense. For such a solution

$$
\psi, \frac{\partial \psi}{\partial x}, \frac{\partial \psi}{\partial y} \text { and } \frac{\partial \psi}{\partial z} \rightarrow 0 \quad \text { as } r \rightarrow \infty,
$$

which implies that $p$ in (2.4) must be chosen so that $p^{2} U=\beta$. Then the function $Z$ defined on $\Pi$ by

$$
Z(x, y, z)=-\frac{1}{y} \psi(x, y, z)
$$

satisfies

$$
\frac{\partial^{2} Z}{\partial x^{2}}+\frac{\partial^{2} Z}{\partial y^{2}}+\frac{2}{y} \frac{\partial Z}{\partial y}+\frac{\partial^{2} Z}{\partial z^{2}}+f(Z)=0
$$

on $\Pi$ in the distributional sense, where

$$
f(Z)= \begin{cases}-p^{2} Z & \text { for } Z \leqslant U \\ k^{2}(Z-U)-\beta & \text { for } Z \geqslant U\end{cases}
$$

Equation (3.3) can be written as

$$
\Delta_{5} Z+f(Z)=0
$$

on $\mathbf{R}^{5}$ by considering $Z$ as a function defined on $\mathbf{R}^{5}$ and $\Delta_{5}$ the 5-dimensional Laplacian in terms of the Cartesian coordinates $x_{1}=x, x_{2}=z$ and the spherical coordinates $y=\sqrt{x_{3}^{2}+x_{4}^{2}+x_{5}^{2}}, \theta, \phi$ with $Z$ independent of $\theta$ and $\phi$. Then

LEMMA 1. $Z \in W^{1,2}\left(\mathbf{R}^{5}\right)$.

This holds because

$$
\begin{aligned}
\int_{\mathbf{R}^{s}} Z^{2} d x_{1} \cdots d x_{5} \\
\quad=\int_{-\infty}^{\infty} \int_{-\infty}^{\infty}\left\{\int_{0}^{\infty} \int_{-\pi / 2}^{\pi / 2} \int_{-\pi}^{\pi} Z^{2} y^{2} \sin \theta d \phi d \theta d y\right\} d x d z \\
\quad=4 \pi \int_{-\infty}^{\infty} \int_{-\infty}^{\infty} \int_{0}^{\infty} Z^{2} y^{2} d y d x d z=4 \pi \int_{\Pi} \psi^{2} d x d y d z
\end{aligned}
$$


and

$$
\begin{aligned}
\int_{\mathbf{R}^{5}}\left|\nabla_{5} Z\right|^{2} d x_{1} \cdots d x_{5} & \left.\left.+\left(\frac{\partial Z}{\partial z}\right)^{2}\right] y^{2} \sin \theta d \phi d \theta d y\right\} d x d z \\
= & \int_{-\infty}^{\infty} \int_{-\infty}^{\infty}\left\{\int _ { 0 } ^ { \infty } \int _ { - \pi / 2 } ^ { \pi / 2 } \int _ { - \pi } ^ { \pi } \left[\left(\frac{\partial Z}{\partial x}\right)^{2}+\left(\frac{\partial Z}{\partial y}\right)^{2}\right.\right. \\
= & \int_{-\infty}^{\infty} \int_{-\infty}^{\infty}\left\{\int _ { 0 } ^ { \infty } \int _ { - \pi / 2 } ^ { \pi / 2 } \int _ { - \pi } ^ { \pi } \left[\left(\frac{\partial \psi}{\partial x}\right)^{2}+\left(\frac{\partial \psi}{\partial y}\right)^{2}\right.\right. \\
& \left.\left.+\left(\frac{\partial \psi}{\partial z}\right)^{2}\right] \sin \theta d \phi d \theta d y\right\} d x d z \\
= & 4 \pi \int_{-\infty}^{\infty}\left|\nabla_{3} \psi\right|^{2} d x d y d z \\
& -4 \pi \int_{-\infty}^{\infty} \int_{-\infty}^{\infty}\left\{\lim _{y \rightarrow \infty} \frac{1}{y} \psi^{2}-\lim _{y \rightarrow 0^{+}} \frac{1}{y} \psi^{2}\right\} d x d z \\
= & 4 \pi \int_{\Pi}\left|\nabla_{3} \psi\right|^{2} d x d y d z \\
&
\end{aligned}
$$

in view of (3.1) and (3.2), where $\nabla_{n}$ is the $n$-dimensional Cartesian coordinate gradient operator.

As $Z \in W^{1,2}\left(\mathbf{R}^{5}\right)$ it follows by regularity theory that $Z \in C^{1, \alpha}\left(\mathbf{R}^{5}\right)$ for some $0<\alpha<1$. Then

LEMMA 2. Either $Z \equiv 0$ or $Z>0$ everywhere in $\mathbf{R}^{5}$.

To see this, suppose that $Z<0$ on some subset $\Omega$ of $\mathbf{R}^{5}$ with $Z=0$ on $\partial \Omega$. Then

$$
\Delta_{5} Z=p^{2} Z<0
$$

on $\Omega$. If $\Omega$ is bounded, the maximum principle [9] implies that $Z \geqslant 0$, whereas if $\Omega$ is unbounded the Phragmen-Lindelöf principle [9] implies that $Z \geqslant 0$. These are both contradictions, so $Z \geqslant 0$ must hold everywhere on $\mathbf{R}^{5}$. Now suppose that $Z=0$ at some point $x_{0}$ and $0<Z<U$ at another point $x_{1}$. Let $B$ be a bounded region on which $0 \leqslant Z \leqslant U$ containing both $x_{0}$ and $x_{1}$ with $x_{0}$ in its interior. Then

$$
\Delta_{5} Z-p^{2} Z=0
$$


on $B$ and $Z$ attains its minimum value of 0 in the interior of $B$, so by the minimum principle [9] $Z \equiv 0$ everywhere on $B$, which is a contradiction. Hence either $Z \equiv 0$ everywhere on $\mathbf{R}^{5}$ or $Z>0$ everywhere on $\mathbf{R}^{5}$. In what follows only the nontrivial solutions will be considered.

Now the function $f$ in (3.4) can be written as the sum $f=f_{1}+f_{2}$, where

$$
f_{1}(Z)=-p^{2} Z
$$

and

$$
f_{2}(Z)= \begin{cases}0 & \text { for } Z \leqslant U \\ \left(k^{2}+p^{2}\right)(Z-U) & \text { for } Z \geqslant U .\end{cases}
$$

This function is continuously differentiable in a neighbourhood of $Z=0$, with $f_{1}$ continuously differentiable and with $f_{2}$ piecewise continuously differentiable and nondecreasing.

The following theorem of Amick and Fraenkel [1], which we use with $n=5$, can now be applied. It is a generalization to weak solutions and piecewise differentiable functions $f$ of a theorem of Gidas, Ni and Nirenberg [4], which is proved by means of maximum principles and domain folding arguments.

THeOREM (Amick and Fraenkel). Let $f:[0, \infty) \rightarrow \mathbf{R}$ be continuously differentiable at and near 0 , with $f=f_{1}+f_{2}$ where $f_{1} \in C^{1}[0, \infty)$ and $f_{2}$ piecewise continuous and nondecreasing on $[0, \infty)$ with a finite number of jumps.

Let $Z>0$ be a $C^{1}\left(\mathbf{R}^{n}\right)$ solution of

$$
\Delta_{n} Z+f(Z)=0
$$

in $\mathbf{R}^{n}$ in the distributional sense. Further (with the usual summation notation) suppose that

$$
Z=\frac{1}{|x|^{m}}\left(a_{0}+\frac{a_{j} x_{j}}{|x|^{2}}+\frac{a_{j k} x_{j} x_{k}}{|x|^{4}}+o\left(\frac{1}{|x|^{2}}\right)\right)
$$

with

$$
\frac{\partial Z}{\partial x_{i}}=\frac{-m x_{i}}{|x|^{m+2}}\left(a_{0}+\frac{a_{j} x_{j}}{|x|^{2}}\right)+\frac{a_{i}}{|x|^{m+2}}-\frac{2 x_{i}}{|x|^{m+4}} a_{j} x_{j}+o\left(\frac{1}{|x|^{m+3}}\right)
$$

as $|x| \rightarrow \infty$, for some $m>0$ and $a_{0}>0$.

Then $Z$ is radially symmetric about some point $c$ and $d Z / d \rho<0$ for $\rho=|x-c|$ $>0$.

The Rossby solitary waves considered here are symmetric about the equator, that is about the origin in the steadily moving coordinate system considered in Section 2. Let $\psi$ be an antisymmetric weak solution of (2.3) and (2.4) satisfying 
(3.1) and (3.2), and corresponding to a Rossby solitary wave. Then from the above theorem

$$
\frac{1}{y} \psi=-Z<0
$$

for $y \neq 0$, where $Z$ depends only on $r=\sqrt{x^{2}+y^{2}+z^{2}}$, that is

$$
\frac{1}{y} \psi(x, y, z)=R^{*}(r)
$$

for some negative function $R^{*}$ of $r>0$. Hence

$$
\psi(x, y, z)=y R^{*}(r)=r R^{*}(r) \sin \theta \sin \phi=R(r) \sin \theta \sin \phi
$$

where $R(r)=r R^{*}(r)$. Consequently we have proved

THEOREM. Any weak antisymmetric solution $\psi$ of Berestov's equation (2.3) and (2.4) has the form (3.6), with $R(r)$ as given by Berestov for the 2-layer or 3-layer solitary wave solutions.

An identical result also holds for the two-dimensional solitary Rossby waves of both Larichev and Reznik [9] and Berestov [2]. For these the above argument is carried out in $\mathbf{R}^{4}$ instead of $\mathbf{R}^{5}$ with the $z$ and $\phi$ variables omitted. Then with $y=r \cos \theta$ and $r=\sqrt{x^{2}+y^{2}}$ it follows that

$$
\psi(x, y)=y R^{*}(r)=r R^{*}(r) \sin \theta=R(r) \sin \theta .
$$

In concluding this section it is noted that had attention been restricted to smoother strong solutions rather than the weak solutions considered here, then it would have been possible to conclude the radial symmetry of $Z$ directly from Gidas, $\mathrm{Ni}$ and Nirenberg [4], taking into account their comments on the extendability of their results to functions $f$ which were not everywhere continuously differentiable.

\section{Uniqueness of solutions}

It follows from the previous section that all antisymmetric solitary Rossby wave solutions of (2.3) for a piecewise linear $F$ in the form (2.4), which decrease asymptotically according to some inverse power of the distance from the centre of the wave, and which are at least as smooth as weak solutions, can be separated as in (3.6). Moreover the switch-over curves from one linear form of $F$ to the other occur on circles about the centre of the wave. From Berestov's analysis in Section 2 the unknown radial function $R(r)$ then satisfies different Bessel-like ordinary 
differential equations in different $r$ intervals. The appropriate equations and the radial regions in which they apply depend on whether two- or three-layer solitary wave solutions are being sought. In each case a unique family of solutions is obtained for appropriate parameter values, for example $k, a, p, A$ and $B$ in the two-layer case. The solutions within each family are equivalent, essentially up to a change of scale and depend on the specific choice of positive parameters $k$ and $a$, in the two-layer case, for example, which can be chosen arbitrarily, as long as their product equals a specific positive value. Moreover these are the only possible antisymmetric solitary wave solutions for the given equations. It is in this sense that the explicit solitary Rossby wave solutions of Berestov [2] are unique.

A similar result can also be concluded for the two-dimensional solutions of Larichev and Reznik [7] for two-layer waves and of Berestov [2] for three-layer waves.

It is reiterated that the restriction to antisymmetric solutions here excludes the rider solutions of Flierl et al. [3]. Their existence does not invalidate or contradict the result presented here. Indeed the rider solutions are associated with antisymmetric solutions, so restriction on the possible types of antisymmetric solutions that can occur consequently restricts the possible type of rider solutions that can occur.

\section{Cylindrical vortices}

The well-known explicit solutions for cylindrical vortices can be considered a special case of the two-dimensional solitary Rossby wave solutions of Larichev and Reznik [7], essentially with the rotational effect omitted. They are stated and derived in, for example, Lamb [6; page 245]. The same argument as above can be used to establish their uniqueness. In this case equation (2.3) is replaced by

$$
\Delta_{2} \psi=-\lambda[\psi-U y]_{+}
$$

in $\mathbf{R}^{2}$, where $\lambda$ is a positive constant and $[t]_{+}=(t+|t|) / 2$, with

$$
\psi, \nabla \psi \rightarrow 0 \text { as } r^{2}=x^{2}+y^{2} \rightarrow \infty .
$$

Using $Z=\psi / y$, equation (5.1) transforms to

$$
\frac{\partial^{2} Z}{\partial x^{2}}+\frac{\partial^{2} Z}{\partial y^{2}}+\frac{2}{y} \frac{\partial Z}{\partial y}=-\lambda[Z-U]_{+},
$$

which in turn can be written as

$$
\Delta_{4} Z+\lambda[Z-U]_{+}=0
$$


in $\mathbf{R}^{4}$ with $x_{1}=x$ and cylindrical coordinates $y=\sqrt{x_{2}^{2}+x_{3}^{2}+x_{4}^{2}}, \theta$ and $\phi$. The maximum principle can then be used to show that nontrivial solutions of (5.2) are strictly positive and then the theorem of Amick and Fraenkel to show that they are radially symmetric. The uniqueness of the explicit cylindrical vortex solutions of (5.1) then follows as for that of the solitary Rossby wave solutions. Moreover this result is global in contrast to the earlier local existence result, namely, the absence of secondary bifurcations, of Keady and Norbury [5; Theorem 1.1].

\section{Acknowledgement}

The author would like to thank Dr. Grant Keady for helpful discussions during the preparation of this paper.

\section{References}

[1] L. J. Amick and L. E. Fraenkel, "The uniqueness of Hill's spherical vortex", Arch. Rational Mech. Anal. 92 (1986), 91-119.

[2] A. L. Berestov, "Solitary Rossby waves", Izv. Acad. Sci. USSR Atmospher. Ocean. Phys. 15 (1979), 443-447.

[3] G. R. Flierl, V. D. Larichev, J. C. McWilliams and G. M. Reznik, "The dynamics of baroclinic and barotropic solitary eddies", Dyn. Atmos. Ocean. 5 (1980), 1-41.

[4] B. Gidas, W.-M. Ni and L. Nirenberg, "Symmetry and related properties via the maximum principle", Comm. Math. Phys. 68 (1979), 209-243.

[5] G. Keady and J. Norbury, "A semilinear elliptic eigenvalue problem I", Proc. Roy. Soc. Edinburgh Sect. A 87 (1980), 65-82.

[6] H. Lamb, Hydrodynamics, 6th Edition (Cambridge University Press, Cambridge, 1932).

[7] V. D. Larichev and G. M. Reznik, "On two dimensional solitary Rossby waves", Dokl. Akad. Nauk SSSR 231 (1976), 1077-1079 (Russian).

[8] J. Pedlosky, Geophysical Fluid Dynamics (Springer-Verlag, Berlin, 1979).

[9] M. Protter and H. Weinberger, Maximum Principles in Differential Equations (Prentice-Hall, Englewood Cliffs NJ, 1967). 